\title{
NOTE ON RINGWORM.
}

\author{
By Vet.-Lieut. R. Butler, A.V.D., Aldershot.
}

THE examination of several specimens of hair and epithelium from cases of supposed ringworm has led me to the conclusion that there exists in the horse an affection which, whilst it presents some of the appearances produced by tinea, is, nevertheless, a non-parasitic eruption.

The appearance of the spores of tricophyton is readily recognised when affecting hair sheaths, and although it is possible that the fact of the parasite not being detected may be due to want of accuracy on the part of the present observer, still it is hardly likely that it would be overlooked in numerous and successive trials, and one would rather readily incline to the idea that the cases observed were a local and temporary form of baldness, due to trophic changes over a circumscribed area, and allied to that variety which in man is termed alopecia areata.

The naked-eye appearance is a bald patch varying in diameter from $\frac{1}{2}$-I inch, covered sparely with broken hair, and not accompanied by any inflammatory changes or the formation of crusts.

Microscopically examined, the hairs present but few changes from the normal, but the bulb often has a ragged appearance due to the spreading of its component cells. There is no proliferation of cells or growth enveloping the sheath, such as is commonly observed when the hair is invaded by tricophyton.

The disease appears to be benign and temporary, stimulation immediately causing a fresh growth of hair, but the area involved is noticeable for a considerable period subsequently.

\section{EDITORIAL ARTICLES.}

\section{THE CURABILITY OF TETANUS.}

IN last number of this Journal there appeared a translation of an article by Dr Behring, describing certain experiments regarding tetanus. It was there shown that even such large animals as horses and sheep could be rendered immune against tetanus by a course of treatment with graduated cultures, variously treated, of the tetanus bacillus, and that the blood-serum of animals thus protected could be used to confer immunity against tetanus on small animals. The latest number of the Zeitschrift fiir Hygiene contains a further contribution regarding tetanus, which probably surpasses in interest anything that has yet been published on the same subject. This article is by Dr Kitasato, who, in the latter part of 1890 , and in common with Dr Behring, published the results of experiments bearing on the 